Noname manuscript No.

(will be inserted by the editor)

\title{
Local Minima of Quadratic Functionals and Control of Hydro-Electric Power Stations
}

\author{
M.M. A. Ferreira • A.F. Ribeiro - G.V. Smirnov
}

Received: November 2013 / Accepted: November 2013

\begin{abstract}
In this paper we consider a control problem for a cascade of hydro-electric power stations where some of the stations have reversible turbines. The objective of our work is to optimize the profit of power production satisfying restrictions on the water level in the reservoirs. From mathematical point of view this is a problem of minimization of an infinite-dimensional quadratic functional subject to cone constraints. We obtain sufficient conditions of optimality and illustrate them with some example.
\end{abstract}

Keywords sufficient conditions $\cdot$ optimal control $\cdot$ pure state constraints $\cdot$ maximum principle

\section{Introduction}

Water is becoming a scarce resource and this has impact on how the water is used to produce electric energy. The management of multireservoir systems has attracted the attention of many researchers in different contexts [1]. It is especially important if there is also a possibility of reusing the downstream water in a situation of drought. This may be implemented in modern reversible hydro-electric power stations, associated with reservoirs along a river basin with a cascade structure, where it is possible both to turbine water from upstream to produce electric power and to pump from downstream to refill an upstream reservoir. Here we consider a simplified model (based on $[2,3,4,5]$ ) for a cascade of hydro-electric power stations where some of the stations have reversible turbines. The water level in the reservoirs is subject to some constraints. The problem is considered in the framework of optimal control theory. Its abstract formulation leads us to consideration of local (non-isolated) minima of infinitedimensional quadratic functionals subject to cone constraint. This subject is considered in section 3 . Sufficient conditions of optimality for local minimizers and also for directional minimizers are established. To our knowledge the treatment of sufficient conditions for directional minimizers is knew. Quadratic forms play an important role in the Calculus of Variations [6,7]. Usually principal attention is paid to Legendre condition. Here we consider quadratic forms depending on the trajectories only and not on their derivatives. However, we assume that the trajectories and the derivatives are subject to some geometric constraints. Second order sufficient optimality conditions for optimal control problems have been studied by many other authors; see, for example, $[8,9,10]$ and the references therein. In general these results are deduced under very strict hypothesis or are elaborate and difficult to apply to particular problems. Taking advantage of the particular structure of the problem we prove sufficient conditions of optimality

M.M. A. Ferreira

FEUP, ISR, University of Porto, Portugal

E-mail: mmf@fe.up.pt

A.F. Ribeiro

University of Porto, Portugal

G.V. Smirnov

Centre of Physics, Department of Mathematics and Applications,

University of Minho, Portugal 


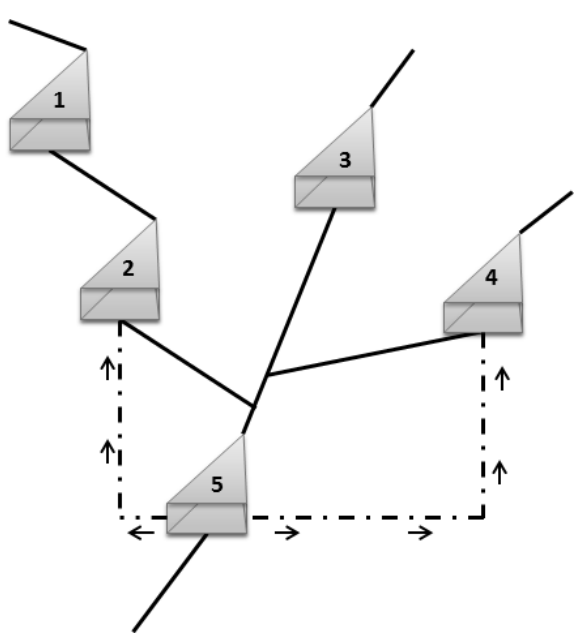

Fig. 1 Cascade of five hydro-electric power stations

allowing one to show that a given solution is optimal in a local sense. Moreover, in the case of one power station we analytically show an improvement of the profit, due to the use of reversible turbines.

\section{Notations and problem statement}

We shall use the following notations: the closure of a set $A$ is denoted by $\operatorname{cl} A$, if $L$ is a linear map then $\operatorname{ker} L$ stands for the kernel of $L$, the graph of a set-valued map $F$ is denoted by graph $F, C([0, T], R)$ represents the space of real continuous functions defined on $[0, T], L_{2}([0, T], R)\left(L_{\infty}([0, T], R)\right)$ represents the space of measurable functions $f:[0, T] \rightarrow R$ whose square is integrable (essential supremum is finite), the space of functions $f:[0, T] \rightarrow R$ of bounded variation is denoted by $B V([0, T], R)$, the space of absolutely continuous functions $f:[0, T] \rightarrow R$ is denoted by $A C([0, T], R)$.

The problem under consideration here is associated to a system of $N$ hydro-electric power stations with a cascade structure. The following picture represents a scheme for a possible cascade of five stations.

The dynamics of water volumes, $V_{k}(t)$, in the reservoirs $k=\overline{1, N}$, is described by the following control system

$$
\dot{V}_{k}(t)=A_{k}-u_{k}(t)+\sum_{m \in M(k)} u_{m}(t), \quad t \in[0, T], \quad k=\overline{1, N},
$$

where $M(k)$ is the set of indices for upstream reservoirs immediately before reservoir $k$.

Set $V(\cdot)=\left(V_{1}(\cdot), \ldots, V_{N}(\cdot)\right)$ and $u(\cdot)=\left(u_{1}(\cdot), \ldots, u_{N}(\cdot)\right)$. The controls $u(t)=\left(u_{1}(t), \ldots, u_{N}(t)\right)$ are the turbined/pumped flows of water for reservoirs at time $t$, and $A_{k}$ are the incoming flows, $k=\overline{1, N}$. The equation (1) is called water balance equation and is present in many references (see, e.g. [11]).

The control variables and the water volumes satisfy the following technical constraints :

$$
V_{k}(0)=V_{k}(T), \quad V_{k}(t) \in\left[V_{k}^{m}, V_{k}^{M}\right], \quad u_{k}(t) \in\left[u_{k}^{m}, u_{k}^{M}\right] .
$$

Here $V_{k}^{m}$ and $V_{k}^{M}, k=\overline{1, N}$, stand for the imposed minimum and maximum water volumes, respectively; $u_{k}^{m}$ and $u_{k}^{M}, k=\overline{1, N}$, are the imposed minimum and maximum turbined/pumped water flows. The objective is to find optimal controls $\hat{u}_{k}(\cdot) \in L_{\infty}([0, T], R)$ and respective volumes $\hat{V}_{k}(\cdot) \in A C([0, T], R)$, that lead to an optimal strategy for the management of water in the system:

$$
\operatorname{maximize} J(u(\cdot), V(\cdot))=\sum_{k=1}^{N} \int_{0}^{T} c(t) u_{k}(t)\left(\frac{V_{k}(t)}{S_{k}}+H_{k}-\frac{V_{j(k)}(t)}{S_{j(k)}}-H_{j(k)}\right) d t .
$$

Here $c(\cdot)$ is the price of the energy, $H_{k}, k=\overline{1, N}$, are the liquid surface elevations, and $S_{k}, k=\overline{1, N}$, are the areas of the reservoirs. The index $j(k)$ is associated to the (unique) downstream reservoir which receives water from reservoir $k$. In $(2)$ the price $c(t)$ is multiplied by an expression representing potential 
energy (for simplicity it is assumed that the reservoirs have cylindric form and that the gravity constant is equal to one). It is assumed that all the potential energy is converted into electric energy.

\section{Local minima of quadratic functionals subject to cone constraints}

In this section we consider the problem of minimization of quadratic functionals subject to cone constraints.

Let $X$ be a Hilbert space, $Y$ and $Z$ be normed spaces, $K \subset Z$ be a closed convex cone, $V: X \rightarrow X$, $A: X \rightarrow Y$, and $C: X \rightarrow Z$, be linear bounded operators, and $v \in X$ be a vector. The operator $V$ is symmetric. Consider the following minimization problem $(P)$ :

$$
\begin{aligned}
& J(x)=\frac{1}{2}\langle x, V x\rangle+\langle v, x\rangle \rightarrow \min , \\
& x \in \Omega=\{x \in X \mid A x=0, \quad-C x \in K\} .
\end{aligned}
$$

We say that $\hat{x} \in \Omega$ is a (local) minimizer point for problem $(P)$ if there exists $\epsilon>0$ such that $J(x) \geq J(\hat{x})$, for all $x \in \Omega \cap\left(\hat{x}+\epsilon B_{X}\right)$.

In this work we will also use the concept of directional minimizer. The point $\hat{x} \in \Omega$ is a directional minimizer point if for all $\bar{x}$ satisfying $\hat{x}+h \bar{x} \in \Omega$ for $h \in\left[0, h_{\bar{x}}\right]$, where $h_{\bar{x}}$ is some positive constant, there exists $\epsilon_{\bar{x}}>0$ such that $J(\hat{x}+h \bar{x}) \geq J(\hat{x})$ for all $h \in\left[0, \epsilon_{\bar{x}}\right]$.

Our aim is to deduce sufficient conditions assuring that zero is a local minimizer for problem $(P)$. Set $L=\operatorname{ker} A \cap \operatorname{ker} C$. Assume that the following conditions are satisfied:

1. $\langle p, V p\rangle \geq 0, p \in L$;

2. there exist $y^{*} \in Y^{*}$ and $z^{*} \in Z^{*}$ such that $A^{*} y^{*}+C^{*} z^{*}+v=0$.

Note that the classical sufficient conditions of optimality in the general mathematical programming problem (see, [12], e.g.) involve the inequality $\langle p, V p\rangle \geq\left(\right.$ const) $|p|^{2}, p \in L$. This guarantees that zero is an isolated local minimizer. In this work, we deal with non-isolated minima and, as a consequence, need a weaker condition. The non-negativity of the quadratic functional $\langle p, V p\rangle$ on the subspace $L$ alone does not guarantee that zero is a local minimum and we shall also assume that one of the following additional conditions is satisfied:

$\left(C_{0}\right)\left\langle z^{*}, C q\right\rangle<0, q \in L^{\perp} \cap \operatorname{ker} A,-C q \in K, q \neq 0$;

$\left(C_{\gamma}\right)$ there exists $\gamma>0$ such that $\left\langle z^{*}, C q\right\rangle \leq-\gamma|q|, q \in L^{\perp} \cap \operatorname{ker} A,-C q \in K$.

Lemma 3.1 Let $x=p+q \in \operatorname{ker} A$, where $p \in L$ and $q \in L^{\perp}$. Then the following inequality holds:

$$
J(x) \geq-\left\langle z^{*}, C q\right\rangle+\langle q, V p\rangle+\frac{1}{2}\langle q, V q\rangle .
$$

Proof. Indeed, we have

$$
J(x)=\langle x, v\rangle+\frac{1}{2}\langle x, V x\rangle .
$$

From this and condition 2 we obtain

$$
J(x)=-\left\langle z^{*}, C q\right\rangle+\langle q, V p\rangle+\frac{1}{2}\langle p, V p\rangle+\frac{1}{2}\langle q, V q\rangle .
$$

Condition 1 implies

$$
J(x) \geq-\left\langle z^{*}, C q\right\rangle+\langle q, V p\rangle+\frac{1}{2}\langle q, V q\rangle .
$$

Proposition 3.1 Assume that condition $\left(C_{0}\right)$ is satisfied, then for all $x \in \operatorname{ker} A$ and $-C x \in K$ there exists $\epsilon>0$ such that the inequality $J(t x) \geq 0$ holds, whenever $t \in[0, \epsilon]$, i.e., 0 is a local directional minimizer.

Proof. From Lemma 3.1 and condition $\left(C_{0}\right)$ we have

$$
J(t x) \geq-t\left\langle z^{*}, C q\right\rangle+t^{2}\langle q, V p\rangle+\frac{t^{2}}{2}\langle q, V q\rangle>0,
$$

whenever $t>0$ is sufficiently small. 
Proposition 3.2 Assume that condition $\left(C_{\gamma}\right)$ is satisfied, then for all $x \in \operatorname{ker} A$ and $-C x \in K$ there exists $\epsilon>0$ such that the inequality $J(x) \geq 0$ holds, whenever $|x| \leq \epsilon$, i.e., 0 is a local minimizer.

Proof. From Lemma 3.1 and condition $\left(C_{\gamma}\right)$ we have

$$
\begin{gathered}
J(x) \geq-\left\langle z^{*}, C q\right\rangle+\langle q, V p\rangle+\frac{1}{2}\langle q, V q\rangle \\
\geq|q|\left(\gamma-|V|\left(|p|+\frac{|q|}{2}\right)\right)>0,
\end{gathered}
$$

whenever $|x|$ is sufficiently small.

Example 3.1 There are directional minimizers that are not minimizers.

Proof. Let $X=L_{2}([0,1], R)$. Consider the problem

$$
\begin{aligned}
& J(x(\cdot))=-\int_{0}^{1} x(s) d s-\int_{0}^{1} x^{2}(s) d s \rightarrow \min , \\
& x(s) \leq 0 .
\end{aligned}
$$

Let $Z=X, K=\left\{z(\cdot) \in L_{2}([0,1], R) \mid z(s) \geq 0, s \in[0,1]\right\}, A=0, C=I$, and $L=\{0\}$. Condition $\left(C_{0}\right)$ is satisfied with $z^{*} \equiv 1$. By Proposition 3.1 zero is a local directional minimizer. Consider the sequence

$$
x_{n}(s)= \begin{cases}-\sqrt{n}, & s \in[0,1 / n] \\ 0, & t \in] 1 / n, 1]\end{cases}
$$

Obviously, $\left|x_{n}(\cdot)\right|_{L_{2}}=1$ and $J\left(t x_{n}(\cdot)\right)=t\left(\frac{1}{\sqrt{n}}-t\right) \geq 0$, only if $t \leq \frac{1}{\sqrt{n}}$.

Lemma 3.2 Let $M \subset X$ be a closed subspace, and let $N \subset X$ be a finite-dimensional subspace. Then $\operatorname{dim}\left(M \cap\left(M^{\perp}+N\right)\right)<+\infty$.

Proof. Let $N=\operatorname{Lin}\left\{e_{1}, \ldots, e_{n}\right\}$. Denote by $\pi_{M}(y)$ the orthogonal projection of $y \in X$ onto $M$. Set $p_{i}=\pi_{M}\left(e_{i}\right), i=\overline{1, n}$. Consider $x \in M \cap\left(M^{\perp}+N\right)$. Then there exist $\xi_{1}, \ldots, \xi_{n} \in R$ and $q \in M^{\perp}$ such that

$$
x=q+\sum_{i=1}^{n} \xi_{i} e_{i} .
$$

Since

$$
x=\pi_{M}(x)=\sum_{i=1}^{n} \xi_{i} \pi_{M}\left(e_{i}\right)=\sum_{i=1}^{n} \xi_{i} p_{i},
$$

we see that any $x \in M \cap\left(M^{\perp}+N\right)$ is a linear combination of vectors $p_{1}, \ldots, p_{n}$.

Proposition 3.3 Assume that $Z=R^{n}, K=R_{+}^{n}$, and $z^{*}>0$. Then condition $\left(C_{\gamma}\right)$ is satisfied.

Proof. Since $(\operatorname{ker} C)^{\perp}=\mathrm{im} C^{*}$ is a finite-dimensional subspace, from Lemma 3.2 we see that the subspace

$$
\operatorname{ker} A \cap L^{\perp}=\operatorname{ker} A \cap \operatorname{cl}\left((\operatorname{ker} A)^{\perp}+(\operatorname{ker} C)^{\perp}\right)=\operatorname{ker} A \cap\left((\operatorname{ker} A)^{\perp}+(\operatorname{ker} C)^{\perp}\right)
$$

is finite-dimensional. Suppose that there exists a sequence $q_{j} \in \operatorname{ker} A \cap L^{\perp},-C q_{j} \in K,\left|q_{j}\right|=1$ such that $\left\langle z^{*}, C q_{j}\right\rangle \uparrow 0$. Without loss of generality $q_{j}$ converges to a vector $q_{0}$. Obviously $q_{0} \in \operatorname{ker} A \cap L^{\perp}$, $-C q_{0} \in K,\left\langle z^{*}, C q_{0}\right\rangle=0$, and $\left|q_{0}\right|=1$. Since $z^{*}>0$, we have $C q_{0}=0$, i.e., $q_{0} \in \operatorname{ker} A \cap \operatorname{ker} C=L$. Therefore we have $q_{0} \in L \cap L^{\perp}=\{0\}$, a contradiction.

Consider functionals $z_{j}^{*} \in K^{*}, j=\overline{1, n}$. Set $\xi_{j}(x)=\left\langle C^{*} z_{j}^{*}, x\right\rangle$ and

$$
\xi(x)=\left(\xi_{1}(x), \ldots, \xi_{n}(x)\right) .
$$


Assume that $Y=R^{m}$ and that the problem has the following special form:

$$
\begin{aligned}
& J(x)=\frac{1}{2}\langle\xi(x), V \xi(x)\rangle+\langle v, \xi(x)\rangle \rightarrow \min , \\
& \Lambda \xi(x)=0 \\
& -C x \in K
\end{aligned}
$$

Consider an auxiliary finite-dimensional majorant problem

$$
\begin{aligned}
& J(x)=\frac{1}{2}\langle\xi, V \xi\rangle+\langle v, \xi\rangle \rightarrow \min , \\
& \Lambda \xi=0 \\
& \xi \leq 0 .
\end{aligned}
$$

Proposition 3.4 Assume that the following conditions are satisfied:

1. $\langle p, V p\rangle \geq 0, p \in \operatorname{ker} A$,

2. there exist $y^{*} \in R^{m}$ and $z^{*} \in R^{n}$ such that $\Lambda^{*} y^{*}+z^{*}+v=0$ and $z^{*}>0$.

Then $\xi=0$ is a local minimizer for the auxiliary problem, and $x=0$ is a local minimizer for the original problem.

Proof. From Proposition 3.3 we see that $\xi=0$ is a local minimizer for the auxiliary problem. Let $x$ be an admissible point. If the norm of $x$ is sufficiently small, then $|\xi(x)|$ is also small. Moreover, the inclusion $-C x \in K$, implies the inequality $\xi(x) \leq 0$. Therefore $\xi(x)$ is an admissible point for the auxiliary problem and $J(x) \geq 0$.

Example 3.2 Consider the problem

$$
\begin{aligned}
& \int_{0}^{1} x(t) d t-\left(\int_{0}^{1} x(t) d t\right)^{2} \rightarrow \min \\
& x(t) \geq 0 .
\end{aligned}
$$

Zero is a local minimizer.

Proof. Set $X=Z=L_{2}([0,1], R), C=I, z^{*}=1$, and

$$
\xi(x)=\int_{0}^{1} x(t) d t
$$

Obviously $\xi=0$ is a solution to the problem

$$
\begin{aligned}
& \xi-\xi^{2} \rightarrow \min , \\
& \xi \geq 0 .
\end{aligned}
$$

We shall deal with the problem

$$
\begin{aligned}
& J(x)+\langle g, x\rangle \rightarrow \min , \\
& x \in \Omega .
\end{aligned}
$$

Set $B_{X}=\{x \in X|| x \mid \leq 1\}$

Proposition 3.5 Assume that there exist $\epsilon>0, y^{*} \in Y^{*}$ and $z^{*} \in K^{*}$ such that

1. $A^{*} y^{*}+C^{*} z^{*}+g=0$,

2. $J(x) \geq 0, x \in \Omega \cap \epsilon B_{X}$.

Then $J(x)+\langle g, x\rangle \geq 0$ whenever $x \in \Omega \cap \epsilon B_{X}$. 
Proof. Indeed, if $x \in \Omega \cap \epsilon B_{X}$, then we have

$$
J(x)+\langle g, x\rangle \geq\langle g, x\rangle=-\left\langle C x, z^{*}\right\rangle \geq 0 .
$$

The second condition can be deduced from Proposition 3.4, for example. Indeed, consider the problem

$$
\begin{aligned}
& I(x)=\frac{1}{2}\langle\xi(x), V \xi(x)\rangle+\langle v, \xi(x)\rangle+\langle g, x\rangle \rightarrow \min , \\
& \Lambda \xi(x)=0, \\
& A x=0, \\
& -C x \in K .
\end{aligned}
$$

The following result is an immediate consequence of Propositions 3.4 and 3.5

Proposition 3.6 Assume that there exist $\epsilon>0, y^{*} \in Y^{*}$, and $z^{*} \subset K^{*}$ such that

1. $A^{*} y^{*}+C^{*} z^{*}+g=0$,

2. condition of Proposition 3.4 are satisfied.

Then $I(x) \geq 0$ whenever $x \in \Omega \cap \epsilon B_{X}$.

The following example shows that if the second condition of Proposition 3.4 is not satisfied then there can exist local directional minimizers that are not local minimizers.

Example 3.3 Consider the problem

$$
\begin{aligned}
& \int_{0}^{1} \phi(s) d s-\phi^{2}(1) \rightarrow \min \\
& \dot{\phi}=u, \quad \phi(0)=0 \\
& \phi(s) \geq 0 .
\end{aligned}
$$

Zero is a local directional minimizer but not a local minimizer.

Proof. The problem can be written in the following form:

$$
\begin{aligned}
& J(x(\cdot))=\int_{0}^{1} \int_{0}^{s} x(r) d r d s-\left(\int_{0}^{1} x(s) d s\right)^{2} \rightarrow \min \\
& \int_{0}^{s} x(r) d r \geq 0 .
\end{aligned}
$$

Here $X=L_{2}([0,1], R), Z=C([0,1], R), Z^{*}=B V([0,1], R), A=0, C=-\int_{0}^{s}, K=\{z(\cdot) \in$ $C([0,1], R) \mid z(s) \geq 0, s \in[0,1]\}, L=\{0\}$, and condition $\left(C_{0}\right)$ is satisfied with $z^{*}=d \mu(s)=d s$. Indeed, if

$$
\int_{0}^{s} q(r) d r \geq 0 \text { and } q(\cdot) \neq 0, \text { then } \exists s \in[0,1]: \int_{0}^{s} q(r) d r>0,
$$

because

$$
\int_{0}^{s} q(r) d r=0, \quad s \in[0,1]
$$

implies $q(\cdot)=0$. By Proposition 3.1 zero is a local directional minimizer. Consider the sequence

$$
x_{n}(s)= \begin{cases}0, & t \in[0,1-1 / n[ \\ \sqrt{n}, & s \in[1-1 / n, 1]\end{cases}
$$

Obviously, $\left|x_{n}(\cdot)\right|_{L_{2}}=1$ and $J\left(t x_{n}(\cdot)\right)=\frac{t}{n}\left(\frac{1}{2 \sqrt{n}}-t\right) \geq 0$, only if $t \leq \frac{1}{2 \sqrt{n}} . \square$ 


\section{Sufficient conditions of optimality for control of hydro-electric power stations}

In this section, motivated by the previous considerations, we deduce sufficient conditions of optimality for control of hydro-electric power stations.

Using (1) the cost function can be written as

$$
\begin{aligned}
J(u(\cdot), V(\cdot)) & =\sum_{k=1}^{N} \int_{0}^{T} c(t)\left(-\dot{V}_{k}(t)+A_{k}+\sum_{m \in M(k)} u_{m}(t)\right) \\
& \times\left(\frac{V_{k}(t)}{S_{k}}+H_{k}-\frac{V_{j(k)}(t)}{S_{j(k)}}-H_{j(k)}\right) d t .
\end{aligned}
$$

Lemma 4.1 The following equality holds:

$$
\int_{0}^{T} \sum_{k=1}^{N} c(t)\left(\frac{V_{k}(t)}{S_{k}} \sum_{m \in M(k)} u_{m}(t)-u_{k}(t) \frac{V_{j(k)}(t)}{S_{j(k)}}\right) d t=0 .
$$

Proof. If $m \in M(k)$ then $k \in j(m)$. Moreover $j(k)$ is empty or has only one element. Therefore we have

$$
\begin{aligned}
& \int_{0}^{T} \sum_{k=1}^{N} c(t)\left(\frac{V_{k}(t)}{S_{k}} \sum_{m \in M(k)} u_{m}(t)-u_{k}(t) \frac{V_{j(k)}(t)}{S_{j(k)}}\right) d t \\
= & \int_{0}^{T} c(t)\left(\sum_{m=1}^{N} \sum_{k \in j(m)} \frac{V_{k}(t)}{S_{k}} u_{m}(t)-\sum_{k=1}^{N} \frac{V_{j(k)}(t)}{S_{j(k)}} u_{k}(t)\right) d t \\
= & \int_{0}^{T} c(t)\left(\sum_{k=1}^{N} \sum_{m \in j(k)} \frac{V_{m}(t)}{S_{m}} u_{k}(t)-\sum_{k=1}^{N} \frac{V_{j(k)}(t)}{S_{j(k)}} u_{k}(t)\right) d t \\
= & \int_{0}^{T} c(t)\left(\sum_{k=1}^{N} \frac{V_{j(k)}(t)}{S_{j(k)}} u_{k}(t)-\sum_{k=1}^{N} \frac{V_{j(k)}(t)}{S_{j(k)}} u_{k}(t)\right) d t=0 .
\end{aligned}
$$

We assume that $c(\cdot) \in B V([0, T], R), c(\cdot)$ is right continuous and $c(0)=c(T)$.

Without changing the notation for the cost function $J$, we convert the maximization problem into a minimization one. Integrating (3) by parts and using Lemma 4.1 we obtain the following problem:

$$
\begin{gathered}
\text { minimize } J(u(\cdot), V(\cdot))= \\
=-\sum_{k=1}^{N}\left[\frac{A_{k}}{S_{k}} \int_{0}^{T} c(t) V_{k}(t) d t+\left(H_{k}-H_{j(k)}\right) \int_{(0, T]}\left(V_{k}(t)+\sum_{m \in \mathcal{M}(k)} V_{m}(t)\right) d c(t)\right. \\
\left.+\frac{1}{2 S_{k}} \int_{(0, T]} V_{k}^{2}(t) d c(t)\right], \\
\dot{V}_{k}(t)=A_{k}-u_{k}(t)+\sum_{m \in M(k)} u_{m}(t), \\
V_{k}(0)=V_{k}(T), \quad V_{k}(t) \in\left[V_{k}^{m}, V_{k}^{M}\right], \quad u_{k}(t) \in\left[u_{k}^{m}, u_{k}^{M}\right] .
\end{gathered}
$$

Let $\mathcal{M}(k)$ be the set containing the indices corresponding to all upstream reservoirs appearing in cascade before reservoir $k$.

First we deduce sufficient conditions for local directional minima. 
Theorem 4.1 Let $\left(\hat{u}_{k}(\cdot), \hat{V}_{k}(\cdot)\right), k=\overline{1, N}$, be a control process. Assume that the following conditions are satisfied:

1. there exist right continuous functions $p_{k}(\cdot) \in B V([0, T], R)$ and $\mu_{k}(\cdot) \in B V([0, T], R), k=\overline{1, N}$, satisfying

$$
\begin{gathered}
d p_{k}(t)=-\frac{A_{k}}{S_{k}} c(t) d t-\left(H_{k}-H_{j(k)}\right) d c(t) \\
-\sum_{l \in \mathcal{M}^{-1}(k)}\left(H_{l}-H_{j(l)}\right) d c(t)-\frac{\hat{V}_{k}(t)}{S_{k}} d c(t)+d \mu_{k}(t), \\
p_{k}(0)=p_{k}(T)
\end{gathered}
$$

2. the equality

$$
\begin{gathered}
\max _{u_{k} \in\left[u_{k}^{m}, u_{k}^{M}\right], k=\overline{1, N}} \sum_{k=1}^{N} p_{k}(t)\left(-u_{k}+\sum_{m \in M(k)} u_{m}\right) \\
=\sum_{k=1}^{N} p_{k}(t)\left(-\hat{u}_{k}(t)+\sum_{m \in M(k)} \hat{u}_{m}(t)\right),
\end{gathered}
$$

holds;

3. the functions $\mu_{k}(\cdot), k=\overline{1, N}$, satisfy the inequalities

$$
\begin{gathered}
d \mu_{k}(t) \leq 0, \quad \text { if } \quad \hat{V}_{k}(t)=V_{k}^{m} ; \quad d \mu_{k}(t) \geq 0, \quad \text { if } \quad \hat{V}_{k}(t)=V_{k}^{M} ; \\
\left.d \mu_{k}(t)=0, \quad \text { if } \quad \hat{V}_{k}(t) \in\right] V_{k}^{m}, V_{k}^{M}[;
\end{gathered}
$$

4. if $d c(t)>0$, then the functions $\mu_{k}(\cdot), k=\overline{1, N}$, satisfy the inequalities

$$
d \mu_{k}(t)<0, \quad \text { if } \quad \hat{V}_{k}(t)=V_{k}^{m} ; \quad d \mu_{k}(t)>0, \quad \text { if } \quad \hat{V}_{k}(t)=V_{k}^{M} ;
$$

5. if $\hat{V}(t) \in] V^{m}, V^{M}[$, then $d c(t) \leq 0$.

Then $J(\hat{u}(\cdot)+h \bar{u}(\cdot), \hat{V}(\cdot)+h \bar{V}(\cdot)) \geq J(\hat{u}(\cdot), \hat{V}(\cdot))$ wherever $\left(\hat{u}_{k}(\cdot)+\bar{u}_{k}(\cdot), \hat{V}_{k}(\cdot)+\bar{V}_{k}(\cdot)\right), k=\overline{1, N}$, is an admissible process and $h>0$ is sufficiently small.

Note. In equation (4) we use the notation $d \nu(t)=f(t) d \varphi(t)$ to express the relationship $\nu(t)-\nu(0)=$ $\int_{(0, t]} f(t) d \varphi(t)$, this integral being a Lebesgue-Stieltjes integral. Also in 3., 4. and 5 . the conditions $d \nu(t) \geq 0(\leq 0,=0)$, for $t \in E$, mean that $\int_{E} f(t) d \nu(t) \geq 0(\leq 0,=0)$ for every nonnegative continuous function $f$.

Proof. Let $\left(\hat{u}_{k}(\cdot)+\bar{u}_{k}(\cdot), \hat{V}_{k}(\cdot)+\bar{V}_{k}(\cdot)\right), k=\overline{1, N}$, be an admissible process, and let $h>0$ be sufficiently small. Then we have

$$
\begin{gathered}
\Delta J=J(\hat{u}(\cdot)+h \bar{u}(\cdot), \hat{V}(\cdot)+h \bar{V}(\cdot))-J(\hat{u}(\cdot), \hat{V}(\cdot)) \\
=-\sum_{k=1}^{N}\left[h \int_{0}^{T} \frac{A_{k}}{S_{k}} c(t) \bar{V}_{k}(t) d t+h \int_{(0, T]}\left(\left(H_{k}-H_{j(k)}\right)+\frac{\hat{V}_{k}(t)}{S_{k}}\right) \bar{V}_{k}(t) d c(t)\right. \\
\left.+h \sum_{m \in \mathcal{M}(k)}\left(H_{k}-H_{j(k)}\right) \int_{(0, T]} \bar{V}_{m}(t) d c(t)+\frac{h^{2}}{2 S_{k}} \int_{(0, T]} \bar{V}_{k}^{2}(t) d c(t)\right] .
\end{gathered}
$$

Using (4) we get

$$
\Delta J=\sum_{k=1}^{N}\left[h \left(\int_{(0, T]} \bar{V}_{k}(t) d p_{k}(t)+\sum_{l \in \mathcal{M}^{-1}(k)}\left(H_{l}-H_{j(l)}\right) \int_{(0, T]} \bar{V}_{k}(t) d c(t)\right.\right.
$$


Observe that

$$
\begin{gathered}
\left.-\int_{(0, T]} \bar{V}_{k}(t) d \mu_{k}(t)\right)-h \sum_{m \in \mathcal{M}(k)}\left(H_{k}-H_{j(k)}\right) \int_{(0, T]} \bar{V}_{m}(t) d c(t) \\
\left.-\frac{h^{2}}{2 S_{k}} \int_{(0, T]} \bar{V}_{k}^{2}(t) d c(t)\right]
\end{gathered}
$$

$$
\sum_{k=1}^{N} \sum_{l \in \mathcal{M}^{-1}(k)}=\sum_{(l, k) \in \operatorname{grph} \mathcal{M}}=\sum_{l=1}^{N} \sum_{k \in \mathcal{M}(l)}
$$

From this we obtain

$$
\Delta J=\sum_{k=1}^{N}\left[h\left(\int_{(0, T]} \bar{V}_{k}(t) d p_{k}(t)-\int_{(0, T]} \bar{V}_{k}(t) d \mu_{k}(t)\right)-\frac{h^{2}}{2 S_{k}} \int_{(0, T]} \bar{V}_{k}^{2}(t) d c(t)\right] .
$$

Integrating by parts and using periodicity conditions we get

$$
\begin{gathered}
\Delta J=\sum_{k=1}^{N}\left[h \int_{0}^{T} p_{k}(t)\left(\bar{u}_{k}(t)-\sum_{m \in M(k)} \bar{u}_{m}(t)\right) d t\right. \\
\left.-h \int_{(0, T]} \bar{V}_{k}(t) d \mu_{k}(t)-\frac{h^{2}}{2 S_{k}} \int_{(0, T]} \bar{V}_{k}^{2}(t) d c(t)\right] \geq 0 .
\end{gathered}
$$

Under some additional conditions on the structure of the problem we can prove sufficient conditions for local minima. Consider a partition of the interval $[0, T], 0=\tau_{0}<\tau_{1}<\ldots<\tau_{Q}=T$. Assume that the price is a piecewise constant function:

$$
c(t)=c_{q}, \quad t \in\left[\tau_{q}, \tau_{q+1}[, \quad q=\overline{0, Q-1}\right.
$$

(We set $c_{Q}=c_{0}$.)

Theorem 4.2 Let $\left(\hat{u}_{k}(\cdot), \hat{V}_{k}(\cdot)\right), k=\overline{1, N}$, be a control process. Assume that the following conditions are satisfied:

1. there exist right continuous functions $p_{k}(\cdot) \in B V([0, T], R)$ and piecewise absolutely continuous functions $\mu_{k}(\cdot), k=\overline{1, N}$, satisfying

$$
\begin{gathered}
d p_{k}(t)=-\frac{A_{k}}{S_{k}} c(t) d t-\left(H_{k}-H_{j(k)}\right) d c(t) \\
-\sum_{l \in \mathcal{M}^{-1}(k)}\left(H_{l}-H_{j(l)}\right) d c(t)-\frac{\hat{V}_{k}(t)}{S_{k}} d c(t)+d \mu_{k}, \\
p_{k}(0)=p_{k}(T) \\
\mu_{k}(t)=\nu_{k}(t)+\sum_{q=1}^{Q} \delta \mu_{k}\left(\tau_{q}\right) H\left(t-\tau_{q}\right)
\end{gathered}
$$

where $\nu_{k}(\cdot) \in A C([0, T], R)$ and $H(\cdot)$ stands for the Heaviside step function;

2. the equality

$$
\begin{gathered}
\max _{u_{k} \in\left[u_{k}^{m}, u_{k}^{M}\right], k=\overline{1, N}} \sum_{k=1}^{N} p_{k}(t)\left(-u_{k}+\sum_{m \in M(k)} u_{m}\right) \\
=\sum_{k=1}^{N} p_{k}(t)\left(-\hat{u}_{k}(t)+\sum_{m \in M(k)} \hat{u}_{m}(t)\right),
\end{gathered}
$$

holds; 
3. the functions $\nu_{k}(\cdot), k=\overline{1, N}$, satisfy the inequalities

$$
\begin{gathered}
d \nu_{k}(t) \leq 0, \quad \text { if } \quad \hat{V}_{k}(t)=V_{k}^{m}, \quad d \nu_{k}(t) \geq 0, \quad \text { if } \quad \hat{V}_{k}(t)=V_{k}^{M} ; \\
\left.d \nu_{k}(t)=0, \quad \text { if } \quad \hat{V}_{k}(t) \in\right] V_{k}^{m}, V_{k}^{M}[;
\end{gathered}
$$

4. if $c_{q-1}<c_{q}$, for some $q=\overline{0, Q}$ then for all $k=\overline{1, N}$, the inequalities

$$
\Delta \mu_{k}\left(\tau_{q}\right)<0 \text {, if } \hat{V}_{k}\left(\tau_{q}\right)=V_{k}^{m} \quad \text { and } \quad \Delta \mu_{k}\left(\tau_{q}\right)>0 \text {, if } \quad \hat{V}_{k}\left(\tau_{q}\right)=V_{k}^{M},
$$

hold;

5. if $\left.\hat{V}_{k}(t) \in\right] V^{m}, V^{M}[$, then $d c(t) \leq 0$.

Then $J(\hat{u}(\cdot)+\bar{u}(\cdot), \hat{V}(\cdot)+\bar{V}(\cdot)) \geq J(\hat{u}(\cdot), \hat{V}(\cdot))$ wherever $\left(\hat{u}_{k}(\cdot)+\bar{u}_{k}(\cdot), \hat{V}_{k}(\cdot)+\bar{V}_{k}(\cdot)\right), k=\overline{1, N}$, is an admissible process and $\max _{q=\overline{0, Q}, k=\overline{1, N}} \bar{V}_{k}\left(\tau_{q}\right)$ is sufficiently small.

Proof. Let $\left(\hat{u}_{k}(\cdot)+\bar{u}_{k}(\cdot), \hat{V}_{k}(\cdot)+\bar{V}_{k}(\cdot)\right), k=\overline{1, N}$, be an admissible process. Arguing as in the proof of the previous theorem we get

$$
\begin{aligned}
\Delta J= & \sum_{k=1}^{N}\left[\int_{0}^{T} p_{k}(t)\left(\bar{u}_{k}(t)-\sum_{m \in \mathcal{M}(k)} \bar{u}_{m}(t)\right) d t-\int_{(0, T]} \bar{V}_{k}(t) d \nu_{k}(t)\right. \\
& \left.-\sum_{q=0}^{Q} \bar{V}_{k}\left(\tau_{q}\right) \Delta \mu_{k}\left(\tau_{q}\right)-\frac{1}{2 S_{k}} \sum_{q=0}^{Q}\left(c_{q}-c_{q-1}\right) \bar{V}_{k}^{2}\left(\tau_{q}\right)\right] \geq 0,
\end{aligned}
$$

whenever $\max _{q=\overline{0, Q}, k=\overline{1, N}} \bar{V}_{k}\left(\tau_{q}\right)$ is sufficiently small.

Assume that the price $c(t)$ is a $T$-periodic function. We shall show that in this case the optimal process is a $T$-periodic extension of the process optimal on the interval $[0, T]$. Let $S>1$ be an integer. Consider the problem

$$
\begin{gathered}
\operatorname{maximize} J(u(\cdot), V(\cdot))=\sum_{k=1}^{N} \int_{0}^{S T} c(t) u_{k}(t)\left(\frac{V_{k}(t)}{S_{k}}+H_{k}-\frac{V_{j(k)}(t)}{S_{j(k)}}-H_{j(k)}\right) d t . \\
\dot{V}_{k}(t)=A_{k}-u_{k}(t)+\sum_{m \in M(k)} u_{m}(t), \quad t \in[0, S T], \quad k=\overline{1, N}, \\
\left.V_{k}(0)=V_{k}(S T), \quad u_{k}(t) \in\left[V_{k}^{m}, V_{k}^{M}\right], \quad u_{k}^{M}\right] .
\end{gathered}
$$

Theorem 4.3 Let $\left(\hat{u}_{k}(\cdot), \hat{V}_{k}(\cdot)\right), k=\overline{1, N}$, be a control process satisfying conditions of Theorem 4.1 or 4.2 on the interval $[0, T]$. Then its T-periodic continuation to the interval $[0, S T]$ is a local directional minimizer (local minimizer) for the above problem.

Proof. It suffices to take the functions $\mu_{k}(\cdot)$ satisfying the conditions $\mu_{k}(t)=\mu_{k}(t-s T)+\mu_{k}(s T)$, $t \in[s T,(s+1) T], s=\overline{1, S-1}$, and follow the proof of Theorem 4.1 or 4.2 . 


\section{Case of one power station}

Here we analyze the case of a system with one power station. We consider a simplified model for real case systems with reversible turbines like the Alqueva dam in Guadiana river in south of Portugal. The Alqueva dam constitutes one of the largest dams and artificial lakes, $250 \mathrm{~km}^{2}$, in Western Europe.

Consider the case of one power station with single incoming flow $A$. Assume that $u^{m}<0<A<u^{M}$. Let $\tau, c_{1}, c_{2}$ be constants such that $\left.\tau \in\right] 0, T\left[\right.$ and $c_{1}<c_{2}$. Take

$$
c(t)=\left\{\begin{array}{l}
c_{1}, t \in[0, \tau[ \\
c_{2}, t \in[\tau, T[ \\
c_{1}, t=T
\end{array}\right.
$$

A direct method, i.e. with no intervention of multipliers, allow us to exclude the possibility of the optimal solution $\hat{u}(t)=A, \hat{V}(t)=V^{M}$ for the problem. In particular, this implies that the use of reversible turbines always improves the profit.

Let the price be as in (5). Consider the problem $(P)$ :

$$
\text { Minimize } J(u(\cdot), V(\cdot))=-\int_{0}^{T} c(t) u(t)\left(\frac{V(t)}{S}+H\right) d t
$$

or equivalently,

$$
\begin{gathered}
\text { Minimize } J(u(\cdot), V(\cdot))=-\frac{c_{1} A}{S} \int_{0}^{\tau} V(t) d t-\frac{c_{2} A}{S} \int_{\tau}^{T} V(t) d t \\
+H\left(c_{2}-c_{1}\right) V(0)+\frac{1}{2 S}\left(c_{2}-c_{1}\right) V^{2}(0)+H\left(c_{1}-c_{2}\right) V(\tau)+\frac{1}{2 S}\left(c_{1}-c_{2}\right) V^{2}(\tau)
\end{gathered}
$$

subject to

$$
\begin{aligned}
& \dot{V}(t)=A-u(t), \\
& V(0)=V(T), \\
& V(t) \in\left[V^{m}, V^{M}\right], \\
& u(t) \in\left[u^{m}, u^{M}\right] .
\end{aligned}
$$

Lemma 5.1 If $(\hat{u}(\cdot), \hat{V}(\cdot))$ is an optimal process for problem $(P)$ and $\hat{V}(0)=V^{M}$, then $\hat{V}(t)=V^{M}$, for all $t \in[0, T]$.

Proof. Let $(\hat{u}(\cdot), \hat{V}(\cdot))$ be an optimal process for problem $(P)$ and $\hat{V}(0)=V^{M}$. Then for every admissible process $(u(\cdot), V(\cdot))$ satisfying $V(0)=V^{M}$ we have,

$$
\begin{gathered}
J(u(\cdot), V(\cdot))=H\left(c_{2}-c_{1}\right) V^{M}+\frac{1}{2 S}\left(c_{2}-c_{1}\right) V^{M} \\
-\frac{c_{1} A}{S} \int_{0}^{\tau} V(t) d t-\frac{c_{2} A}{S} \int_{\tau}^{T} V(t) d t+H\left(c_{1}-c_{2}\right) V(\tau)+\frac{1}{2 S}\left(c_{1}-c_{2}\right) V^{2}(\tau) \\
\geq H\left(c_{2}-c_{1}\right) V^{M}+\frac{1}{2 S}\left(c_{2}-c_{1}\right) V^{M}-\frac{c_{1} A}{S} \int_{0}^{\tau} V^{M} d t-\frac{c_{2} A}{S} \int_{\tau}^{T} V^{M} d t \\
+H\left(c_{1}-c_{2}\right) V^{M}+\frac{1}{2 S}\left(c_{1}-c_{2}\right)\left(V^{M}\right)^{2} .
\end{gathered}
$$

If $V(t)<V^{M}$ on some subset, the above inequality is strict. Since the inequality is still valid for $(u(\cdot), V(\cdot))=(\hat{u}(\cdot), \hat{V}(\cdot))$ and this process is optimal we obtain $\hat{V}(t)=V^{M}$.

Lemma 5.2 The optimal process $(\hat{u}(\cdot), \hat{V}(\cdot))$ for problem $(P)$ satisfies $\hat{V}(0)<V^{M}$. 
Proof. Assume that $\hat{V}(0)=V^{M}$. Then by Lemma 5.1 we have $\hat{V}(t) \equiv V^{M}$. Moreover $\hat{u}(t) \equiv A$. Consider the family of processes $\left(u_{y}(\cdot), V_{y}(\cdot)\right)$, defined as

$$
u_{y}(t)= \begin{cases}u^{m}, & \text { if } t \in[0, \alpha[ \\ A, & \text { if } t \in[\alpha, \beta[, \\ u^{M}, & \text { if } t \in[\beta, T[,\end{cases}
$$

and

$$
V_{y}(t)= \begin{cases}y+\left(A-u^{m}\right) t, & \text { if } t \in[0, \alpha[, \\ V^{M}, & \text { if } t \in[\alpha, \beta[, \\ V^{M}+\left(A-u^{M}\right)(t-\beta), & \text { if } t \in[\beta, T[,\end{cases}
$$

with

$$
\alpha=\frac{V^{M}-y}{A-u^{m}} \quad \text { and } \quad \beta=T-\frac{V^{M}-y}{u^{M}-A},
$$

where $V^{M}-y>0$ is small enough to satisfy $y>V^{m}$ and $\alpha<\tau<\beta$.

Then we have

$$
\begin{gathered}
J\left(u_{y}(\cdot), V_{y}(\cdot)\right)-J(\hat{u}(\cdot), \hat{V}(\cdot))= \\
=-\frac{c_{1} A}{S}\left(y \alpha+\left(A-u^{m}\right) \frac{\alpha^{2}}{2}-V^{M} \alpha\right)-\frac{c_{2} A}{S}\left(\left(A-u^{M}\right) \frac{(T-\beta)^{2}}{2}\right) \\
+H\left(c_{2}-c_{1}\right)\left(y-V^{M}\right)+\frac{1}{2 S}\left(c_{2}-c_{1}\right)\left(y^{2}-\left(V^{M}\right)^{2}\right) \\
=\frac{c_{1} A}{2 S} \alpha\left(V^{M}-y\right)-\frac{c_{2} A}{S}\left(\left(A-u^{M}\right) \frac{(T-\beta)^{2}}{2}\right) \\
+H\left(c_{2}-c_{1}\right)\left(y-V^{M}\right)+\frac{1}{2 S}\left(c_{2}-c_{1}\right)\left(y^{2}-\left(V^{M}\right)^{2}\right) \\
=\frac{c_{1} A}{2 S} \frac{\left(V^{M}-y\right)^{2}}{A-u^{m}}+\frac{c_{2} A}{2 S} \frac{\left(V^{M}-y\right)^{2}}{u^{M}-A}+H\left(c_{2}-c_{1}\right)\left(y-V^{M}\right)+\frac{1}{2 S}\left(c_{2}-c_{1}\right)\left(y^{2}-\left(V^{M}\right)^{2}\right) \\
=\left(V^{M}-y\right) G(y)
\end{gathered}
$$

where

$$
G(y)=\frac{c_{1} A}{2 S} \frac{V^{M}-y}{A-u^{m}}+\frac{c_{2} A}{2 S} \frac{V^{M}-y}{u^{M}-A}-H\left(c_{2}-c_{1}\right)-\frac{1}{2 S}\left(c_{2}-c_{1}\right)\left(y+V^{M}\right) .
$$

Since $G(y)$ is linear in $y$ and $G\left(V^{M}\right)=-H\left(c_{2}-c_{1}\right)-\frac{1}{S}\left(c_{2}-c_{1}\right) V^{M}<0$, we have

$$
J\left(u_{y}(\cdot), V_{y}(\cdot)\right)<J(\hat{u}(\cdot), \hat{V}(\cdot))
$$

whenever $y<V^{M}$ is close to $V^{M}$, a contradiction.

Theorem 5.1 Let $(\hat{u}(\cdot), \hat{V}(\cdot))$ be an optimal process for problem $(P)$. Then $\hat{u}(t)<0$ on some non null measure set.

Proof. By Lemma 5.2 we have $\hat{V}(0)<V^{M}$. Consider the set of admissible trajectories $V(\cdot)$ satisfying $V(0)=\hat{V}(0)$. The associated cost is

$$
\begin{gathered}
J(u(\cdot), V(\cdot))=H\left(c_{2}-c_{1}\right) \hat{V}(0)+\frac{1}{2 S}\left(c_{2}-c_{1}\right) \hat{V}^{2}(0) \\
-\frac{c_{1} A}{S} \int_{0}^{\tau} V(t) d t-\frac{c_{2} A}{S} \int_{\tau}^{T} V(t) d t+H\left(c_{1}-c_{2}\right) V(\tau)+\frac{1}{2 S}\left(c_{1}-c_{2}\right) V^{2}(\tau) .
\end{gathered}
$$

Suppose that $\hat{u}(t) \geq 0$, a.e. $t \in[0, T]$. Take $\theta=\min \left\{s \mid \hat{V}(s)=\max _{t \in[0, \tau]} \hat{V}(t)\right\}$. Since $c_{2}>c_{1}$, we have $\max _{t \in[0, T]} \hat{V}(t) \geq \hat{V}(0)$ 
Consider the process $(\tilde{u}(\cdot), \tilde{V}(\cdot))$, with $\tilde{V}(0)=\hat{V}(0)$ and

$$
\tilde{u}(t)= \begin{cases}u^{m}, & \text { if } t \in[0, \alpha[ \\ A, & \text { if } t \in[\alpha, \theta[, \\ \hat{u}(t), & \text { if } t \in[\theta, T] .\end{cases}
$$

Here $\alpha$ is chosen to satisfy $\tilde{V}(\alpha)=\hat{V}(\theta)$. Therefore we have $A \theta-\int_{0}^{\theta} \hat{u}(\tau) d \tau=\alpha\left(A-u^{m}\right)$. Hence,

$$
\alpha=\frac{A \theta-\int_{0}^{\theta} \hat{u}(\tau) d \tau}{A-u^{m}} \leq \frac{A \theta}{A-u^{m}}<\theta .
$$

Since $\hat{u}(t) \geq 0$, by definition of $\tilde{u}$ we have $\tilde{V}(t) \geq \hat{V}(t)$ on $[0, \theta]$ and $\tilde{V}(t)=\hat{V}(t)$ on $[\theta, T]$. Comparing cost functions we obtain

$$
J(\tilde{u}(\cdot), \tilde{V}(\cdot))-J(\hat{u}(\cdot), \hat{V}(\cdot))=-\frac{c_{1} A}{S} \int_{0}^{\alpha}(\tilde{V}(t)-\hat{V}(t)) d t-\frac{c_{1} A}{S} \int_{\alpha}^{\theta}(\hat{V}(\theta)-\hat{V}(t)) d t \quad<0,
$$

a contradiction.

Thus we see that the use of reversible turbines improves the profit.

Under an additional condition we can explicitly find an optimal process.

Theorem 5.2 Let $V^{m}<V^{M}-\theta\left(A-u^{m}\right)$, where

$$
\theta=\frac{u^{M}-A}{A-u^{m}}(T-\tau)
$$

Assume that $\theta<\tau$. Then the process $(\hat{u}(\cdot), \hat{V}(\cdot))$, where

$$
\hat{u}(t)=\left\{\begin{array}{l}
u^{m}, t \in[0, \theta] \\
A, \quad t \in] \theta, \tau] \\
\left.\left.u^{M}, t \in\right] \tau, T\right]
\end{array}\right.
$$

and

is optimal.

$$
\hat{V}(t)= \begin{cases}V^{M}+(t-\theta)\left(A-u^{m}\right), & t \in[0, \theta], \\ V^{M}, & t \in] \theta, \tau], \\ V^{M}+(t-\tau)\left(A-u^{M}\right), & t \in] \tau, T],\end{cases}
$$

Proof. Consider also the functions

$$
\mu(t)=\left\{\begin{array}{lr}
0, & t \in[0, \theta[, \\
c_{1}(t-\theta) A / S, & t \in[\theta, \tau[, \\
c_{1}(\tau-\theta) A / S+\Delta \mu, & t \in[\tau, T],
\end{array}\right.
$$

and

where

$$
p(t)= \begin{cases}(\theta-t) c_{1} A / S, & t \in[0, \theta[ \\ 0, & t \in[\theta, \tau[ \\ p_{\tau}-(t-\tau) c_{2} A / S, & t \in[\tau, T[ \\ p(T)=\theta c_{1} A / S, & t=T,\end{cases}
$$

and

$$
\Delta \mu=\theta \frac{c_{1} A}{S}+(T-\tau) \frac{c_{2} A}{S}+\frac{c_{2}-c_{1}}{S}(\hat{V}(\tau)-\hat{V}(0))
$$

$$
p_{\tau}=-\left(c_{2}-c_{1}\right) H-\frac{c_{2}-c_{1}}{S} \hat{V}(\tau)+\Delta \mu .
$$

If $p_{\tau} \leq 0$, then, since $\bar{V}(\tau) \leq 0$ and $\Delta \mu>0$, from Theorem 4.2 we see that $(\hat{u}(\cdot), \hat{V}(\cdot))$ is a local minimizer for the problem. Note that in this case we have

$$
\Delta J \geq-\Delta \mu \bar{V}(\tau)-\frac{c_{2}-c_{1}}{2 S} \bar{V}^{2}(\tau) \geq 0,
$$

whenever $\hat{V}(\cdot)+\bar{V}(\cdot)$ is admissible and $\bar{V}(\tau)$ is sufficiently small. $\square$

Consider an illustrative example. 
Example 5.1 Let $A=1, T=12, \tau=6, c_{1}=2, c_{2}=5, u^{m}=-1, u^{M}=2, V^{m}=3, V^{M}=10$, $S=100$. Then $\hat{V}(0)=4$ and $\theta=3$. The condition $p_{\tau}<0$ is satisfied. The optimal process is shown in Fig. 2.

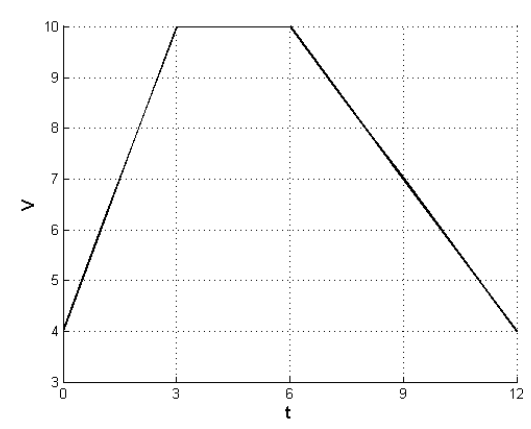

Fig. 2 Optimal process

Note that $\hat{u}(t)=-1, t \in[0,3]$, i.e. the water is pumped. In this way the station accumulates water when the price is low to be used when the price is higher.

\section{Conclusions}

In this work we analysed an optimal control problem modeling a cascade of hydro-electric power stations where some of the stations have reversible turbines and with objective the optimization of the profit of power production. This is a problem of minimization of an infinite-dimensional quadratic functional subject to cone constraints. Results on this subject where deduced. Generally and for the considered problems the minimum point may be not isolated or even it can be only a directional minimizer point. In such cases sufficient conditions of optimality are much more effective when compared to necessary conditions. Besides sufficient conditions of optimality for the problem of minimizing the profit on a cascade of hydro-electric power stations, it was determined the structure of the solution for such problem when the price is periodic. This can be important in real situations. The particular case of one power station was studied in more detail. It was proved that the use of reversible turbines always improves the profit in that case.

Acknowledgements This work has been partially supported by the European Union Seventh Framework Programme [FP7-PEOPLE-2010-ITN] under grant agreement n. 64735-SADCO and FCT project PTDC/EEA$\mathrm{CRO} / 116014 / 2009$.

\section{References}

1. Labadie, J. W.: Optimal Operation of multireservoir systems: state-of-the-art review. J. Water Resour. Plng. and Mgmt. 130(2), pp.93-111 (2004) 1

2. Korobeinikov, A., Kovacec, A., McGuinness, M., Pascoal, M., Pereira, A., Vilela, S.: Optimizing the profit from a complex cascade of hydroelectric stations with recirculating water. Mathematics-in-industry case studies journal. 2, pp.111-133 (2010) 1

3. International Atomic Energy Agency, Valoragua - A model for the optimal operating strategy of mixed hydrothermal generating systems, Users' manual for the mainframe computer version. IAEA computer manual series no. 4, Vienna (1992) 1

4. Liang, R., Hsu, Y.: Hydroelectric generation scheduling using self-organizing feature maps. Electric Power Systems Research. 30(1), pp.1-8 (1994) 1

5. Ribeiro, A. F., Guedes, M. C. M., Smirnov, G. V., Vilela, S.: On the optimal control of a cascade of hydroelectric power stations. Electric Power Systems Research. 88, pp.121-129 (2012) 1

6. Ioffe, A.D. ,Tihomirov, V.M.: Theory of Extremal Problems.North-Holland, Amsterdam, (1979) 1 
7. Arutyunov, A. V.: Optimality Conditions: Abnormal and Degenerate Problems. Kluwer Academic Publishers, (2000) 1

8. Malanowski, K., Maurer, H., Pickenhain, S.: Second-Order Sufficient Conditions for State-Constrained Optimal Control Problems. Journal of Optimization Theory and Applications. 123(3), pp.595-617 (2004) 1

9. Osmolovskii, N., Maurer,H.: Applications to regular and Bang-Bang Control: Second-Order Necessary and Sufficient Optimality Conditions in Calculus of Variations and Optimal Control. SIAM, Philadelphia (2012) 1 10. Hoehener, D.: Variational Approach to Second-Order Optimality Conditions for Control Problema with Pure State Constrains. SIAM, J. Control Optim. 50(3), pp.1139-1173 (2012) 1

11. Mariano, S.J.P., Catalao, J.P.S., Mendes, V.M.F., Ferreira, L.A.F.M.: Profit-Based Short-Term Hydro Scheduling considering Head-Dependent Power Generation. Power Tech, 2007 IEEE Lausanne, pp.1362-1367 (July 2007) 2

12. McCormick, G.: Second order conditions for constrained minima. SIAM, J. Appl. Math. 15(3), pp. 641-652 (1967) 3 